\title{
MANAGEMENT OF COMPLETE TRANSECTION OF DUODENUM AND PARTIAL TRANSECTION OF TRANSVERSE COLON IN BLUNT INJURY ABDOMEN IN A RURAL SETUP
}

\author{
Periasamy Subbiah¹, Gayathre Shanmugam Papu², Ramaswamy Rajendran³, Prithviraj Premkumar 4 \\ 1 Professor, Department of General Surgery, Government Chengalpattu Medical College, Chengalpattu, Tamilnadu. \\ ${ }^{2}$ Associate Professor, Department of General Surgery, Thanjavur Medical College, Thanjavur, Tamilnadu. \\ ${ }^{3}$ Trainee, Department of Surgical Oncology, Madras Medical College, Rajiv Gandhi Government General Hospital, Chennai, Tamilnadu. \\ ${ }^{4}$ Trainee, Department of Surgical Oncology, Madras Medical College, Rajiv Gandhi Government General Hospital, Chennai, Tamilnadu.
}

\section{ABSTRACT}

Complete transection of duodenum and partial transection of transverse colon following blunt injury abdomen and its clinical picture is often obscure and is extremely rare. High index of suspicion on the basis of mechanism of injury is important in early diagnosis. Early intervention in duodenal injuries have improved outcome and if it is more than 24 hours the mortality increased from 11 to $40 \%$. The retroperitoneal location of the duodenum, its proximity to important abdominal structures and organs, its marginal blood supply, the biliary, pancreatic secretion drainage and diagnostic delay of its injuries cause therapeutic difficulties. All these factors create intraoperative dilemmas in the surgical management of duodenal injuries. The management of duodenal traumas remains controversial. We have discussed here a case of complete transection of duodenum and partial transection of transverse colon injury in blunt injury abdomen with the handle bar in a two wheeler accident.

\section{KEYWORDS}

Duodenal Injury, Blunt Injury Abdomen, Tension Free Primary Anastomosis.

HOW TO CITE THIS ARTICLE: Subbiah P, Papu GS, Rajendran R, et al. Management of complete transection of duodenum and partial transection of transverse colon in blunt injury abdomen in a rural setup. J. Evolution Med. Dent. Sci. 2016;5(44):2781-2784, DOI: $10.14260 /$ jemds/2016/649

\section{INTRODUCTION}

The duodenum is injured due to crushing or shearing forces on the abdomen. Isolated duodenal injury comprises $0.1 \%$ of all blunt injury abdomen.

Forty percent of patients with duodenal injuries have other concomitant surgically important intra-abdominal injuries, such as hepatic $(38 \%)$ or pancreatic $(28 \%)$ injuries.[1]

The anatomical location of the duodenum makes diagnosis and treatment of isolated duodenal injury a difficult task. Due to its rarity and subtle clinical features, the diagnosis and management is often delayed. Here we present a case report of complete transection of $3^{\text {rd }}$ part of duodenum along with partial transection of transverse colon following blunt injury abdomen, operative management and the surgical technique have been described and discussed.

\section{CASE REPORT}

33 years old male presented to our emergency department with history of a road traffic accident. Blunt injury to abdomen with the handle bar. Two hours after injury patient developed upper abdominal pain and had 4 episodes of vomiting, which had food particles with progressive distention of abdomen.

On general examination, patient had Pulse rate of 102/minute and Blood pressure of 96/60 mmHg. Abdomen examination findings were tenderness in the epigastric region associated with rebound tenderness and guarding, distended abdomen with sluggish bowel sounds. Per rectal examination revealed soiling with faeces.

Financial or Other, Competing Interest: None.

Submission 18-04-2016, Peer Review 14-05-2016,

Acceptance 18-05-2016, Published 02-06-2016.

Corresponding Author:

Dr. Ramaswamy Rajendran,

New No. 17, Old No. 6,

$3^{\text {rd }}$ Main Road Gandhi Nagar

Adyar, Chennai-600020.

E-mail: drramasamy88@gmail.com

DOI: 10.14260/jemds/2016/649
Patient was stabilized with intravenous fluids. Blood investigations including complete haemogram, renal function test and liver function test done. X-ray chest PA view and X-ray abdomen erect showed air under diaphragm.

CT abdomen revealed free fluid in pelvis and in the right paracolic gutter. On the high index of suspicion, emergency laparotomy was planned. Prophylactic antibiotic was given.

Exploratory laparotomy was done. Around $1200 \mathrm{~mL}$ of blood mixed with peritoneal fluid and partial transection of half of the circumference of the transverse colon were found. The peritoneal fluid was evacuated and the remaining peritoneal fluid was to be bile stained. Whole of jejunum and ileum inspected for any injury and were found to be normal. On suspicion we did Kocherisation of duodenum up to fixed part of jejunum. The third part of the duodenum was found to be completely transected (Figure 1). Pancreas and rest of the abdominal organs were normal.

Contamination of the peritoneum was minimal with healthy cut edge of the duodenum. We performed a primary tension free anastomosis between the duodenum and the jejunum, as it would be more beneficial for the patient. We mobilized the third and fourth part of the duodenum along with the proximal end of jejunum, releasing the duodenum from the superior mesenteric vessel crossover (Figure 2), damaged distal portion of the duodenum and the proximal 3 $\mathrm{cm}$ of the jejunum (DJ Flexure) were resected, so the jejunum could be mobilized well to have access to the third part of the duodenum for a duodeno-jejunostomy (end-to-side anastomosis) after the closure of the cut end of the jejunum (Figure 3). The Ryles tube from the stomach was mobilized down to the duodenum and across the anastomotic site, so that it will act as a stent preventing the stasis of secretions and chances of anastomotic leak.

Partial transected transverse colon edges were trimmed and brought out as loop colostomy. We have not tried for the primary closure of colon since too many anastomoses may jeopardize our diagnosis of site of post-op leak. 
Laparotomy wound was closed with feeding jejunostomy and drain in the sub-hepatic and pelvis region.

Patient was kept on nil per oral for 2 days, feeding started through Feeding Jejunostomy on $3^{\text {rd }}$ post-op day. Patient passed solid stools through colostomy on $5^{\text {th }}$ post-op day. Ryle tube removed on $9^{\text {th }}$ post-op day. Patient started on oral feeds on $9^{\text {th }}$ post-op day. Pelvic drain and sub-hepatic drain removed on $11^{\text {th }}$ post-op day. Patient was doing well and serum amylase levels were normal. Post-operative wound healthy. Patient was discharged on $16^{\text {th }}$ post-op day. Transverse colostomy closure was done in an elective setting after 5 weeks.

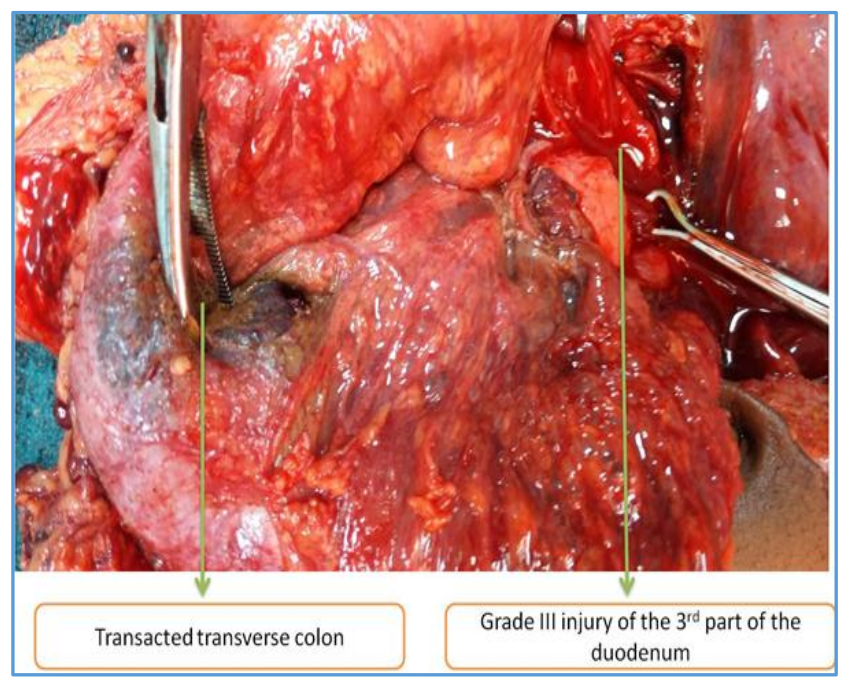

Fig. 1

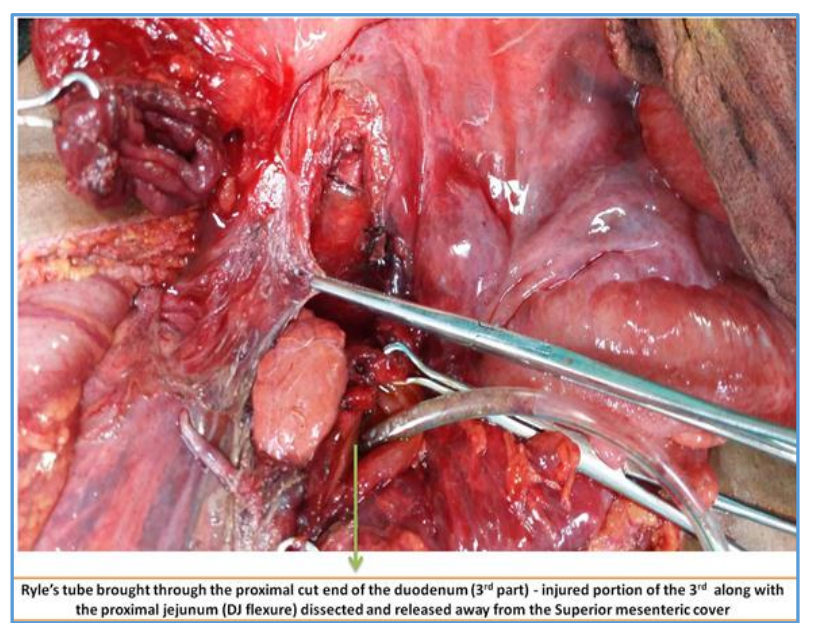

Fig. 2: Ryle's brought through the Proximal Cut End of the Duodenum (3rd part) - Injured Portion of the $3^{\text {rd }}$ along with the Proximal Jejunum (DJ Flexure) Dissected and Released away from the Superior Mesenteric Cover

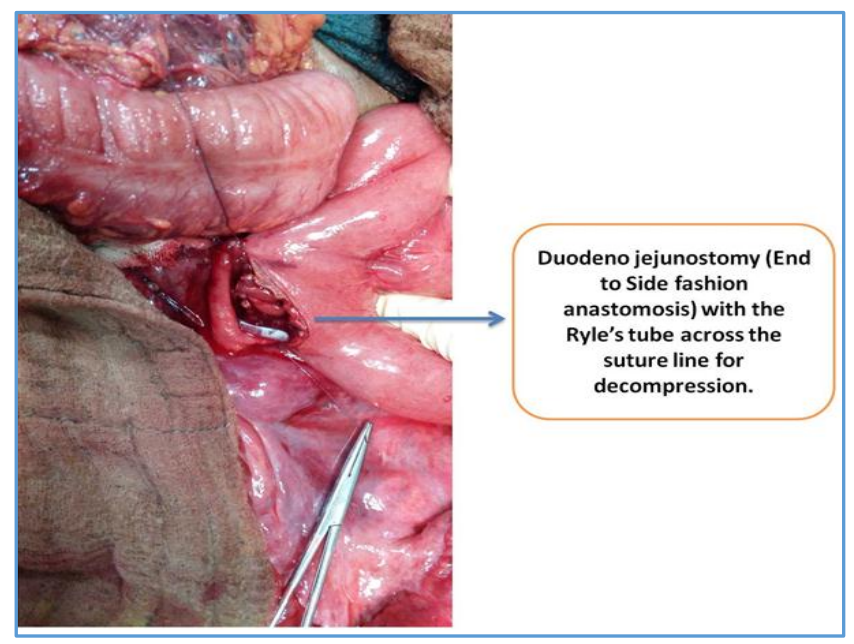

Fig. 3

\section{DISCUSSION}

Duodenal injuries are uncommon because duodenum is in retro peritoneum. The duodenum is mobile only at the pyloric end. There is difficulty in suturing or reflecting the duodenum because it shares the blood supply with pancreas. It is very challenging to the surgeon and failure to manage it properly can result in devastating results. The initial clinical changes in duodenal injuries may be extremely subtle before life threatening peritonitis develops. Mechanism of injury and physical examination is the key in early detection of duodenal injuries.

Thus the following Clinical Signs Detected should be Particularly Emphasized

- Abdominal pain, especially when the right upper quadrant is injured. And if the pain is intensified progressively with apparent peritoneal stimulation and radiation pain to the back, the duodenum is very likely injured.

- $\quad$ Retching or blood stained vomitus.

- Abdominal distension, especially in the upper quadrant with sluggish bowel sounds.

- Anterior sacral crepitus on digital rectal examination.(2)

- Diagnostic Peritoneal Lavage (DPL) has been found to have a low diagnostic sensitivity for duodenal rupture because of the retroperitoneal location of the duodenum. ${ }^{(3)}$

Abdominal plain films, ultrasound scan and CT scan can also help the diagnosis of duodenum injury. Retroperitoneal air, free intra-peritoneal air or other signs such as obliteration of the psoas muscle shadow and scoliosis of the lumbar vertebrae can give a clue of injury.(4)

Although, routine laboratory tests are not helpful in the preoperative diagnosis of duodenal rupture, some authors find that the serum amylase is an important marker. Serum amylase is elevated in $50 \%$ of patients with duodenal or upper gastrointestinal injury.

Explorative laparotomy remains the ultimate diagnostic test if a high degree of suspicion of duodenal injury continues. The explorative procedures should be careful, comprehensive, accurate and quick. 


\section{The Duodenum should be Explored if such Signs Appear}

1. Free gas or fluid looking like bile with undetermined origin;

2. Extraction of intestinal juice or fluid like bile from retroperitoneal haematoma;

3. Periduodenal area, retroperitoneum, mesocolon and the root of mesentery presenting with oedema, haematoma, ecchymosis or crepitus.

4. Instillation of Methylene Blue via NG tube.(5)

It requires a careful detection for an accompanied injury to the pancreas as well as the bile duct and the ampulla, especially when the second and third portion of duodenum is injured.

Scoring Duodenal Injury (American Association for the Surgery of Trauma).(6)

Grade I Haematoma, laceration involvement of a single portion of the duodenum.

Grade II Haematoma, laceration involvement of more than one portion, disruption of $<50 \%$ of the circumference.

Grade III Laceration Disruption of $50 \%-75 \%$ of the circumference of D2; disruption of $50 \%-100 \%$ of the circumference of D1, D3 and D4.

Grade IV Laceration Disruption of $>75 \%$ of the circumference of D2 or involvement of the ampulla or distal common bile duct.

Grade V Laceration, vascular injury Massive disruption of the duodeno-pancreatic complex or devascularisation of the duodenum.

Note-Major variables are involvement of one or more parts of the organ, type of injury (haematoma, laceration or disruption), and involvement of the ampulla and bile duct. The duodenum is divided into the duodenal bulb (D1), descending part (D2), transverse part (D3) and ascending part (D4).

Four basic principles in managing duodenal trauma: Restore intestinal continuity, Decompress the duodenal lumen, Provide wide and external drainage, nutritional support.

For intramural haematoma, which develops in a submucosal or subserosal layers without perforation. Haematoma evacuation without violating the mucosa and bowel wall repair needs to be done carefully. However, controversially others believe that operation will increase the incidence of perforation and to leave the haematoma intact is better. Nasogastric decompression after operation is alone sufficient.(7)

If the range of duodenum injury is less than $50 \%$ of the circumference with regular injury border, adequate blood supply and without serious peritoneal contamination, the injury could be closed transversely and the decompression of duodenum could be achieved by jejunostomy. It is believed that $75 \%-85 \%$ of duodenum injury could be closed primarily and the incidence of duodenal fistula is less than $10 \%$.

If the duodenal injury is more than $50 \%$ of the circumference or scenarios, where primary closure of the defect may narrow the lumen of the bowel or cause tension in the suture line with subsequent breakdown, segmental resection and primary end-to-end duodeno-duodenostomy is advised, especially when the first, second or third part of duodenum is injured.

If a large part of duodenum is non-viable due to injury, tension free suturing of the two ends will be impossible. Surgeries of duodenal diverticulization should be carried out, when a large tissue of the first part of duodenum is lost; it includes closure of the duodenal injury, gastric antrectomy with end-to-side gastrojejunostomy, tube duodenostomy and drainage the area of duodenal repair.(8) The disadvantages of duodenal diverticulization are, it is a time-consuming procedure and hence not recommended in haemodynamically unstable patients or when several other injuries are presented. Otherwise, if such injury is distal to the ampulla of Vater, closure of distal duodenum and Roux-en-Y duodenojejunostomy is the procedure of choice. If the injury happens to the second part of duodenum, because of the limited mobilization of this part, a direct anastomosis of Roux-en-Y over the injury in an end-to-side fashion is done. This method can be also be applied to other parts when the primary anastomosis is impossible.

\section{Indications for a Whipple's Procedure:}

- Massive and uncontrollable bleeding from the head of the pancreas, adjacent vascular structures or both.

- Massive and unreconstructable ductal injury in the head of the pancreas.

\section{Combined Unreconstructable Injuries of the following:}

- Duodenum and head of the pancreas

- Duodenum, head of the pancreas, and common bile duct However, in a study of Asensio and Colleagues which included 170 patients who underwent pancreaticoduodenectomy in 50 reported series, the overall mortality rate reached $33 \%$. So pancreaticoduodenectomy should be applied carefully. (9)

The most serious complication following the treatment of duodenal injury is duodenal fistula with average incidence of $\left.6 \% .{ }^{9}\right)$ Fistula rate of $2.3 \%$ with tube decompression and $11.8 \%$ without.(10) other complications reported with duodenal trauma include intra-abdominal abscess, pancreatitis, duodenal obstruction and bile duct fistula. The overall mortality rate of duodenal injuries remains to be significant with an average incidence of $17 \%$.

\section{CONCLUSION}

Exploratory laparotomy remains the final diagnostic test if a high suspicion of duodenal injury continues after all investigations are applied. Most duodenal injuries can be managed by simple repair. More complicated injuries need more complex operative techniques. There is a high chance of post-operative complications, especially the duodenal fistula and high mortality. Ryle tube decompression and external drainage are necessary and helpful in reducing the complication rates. The peri-operative nutritional support decreases morbidity and enhances post-operative recovery.

We presented this case, not for its rarity but for the management of duodenal injuries in $3^{\text {rd }}$ part with duodenojejunal anastomosis without tension in a primary setting. Postoperative period was uneventful and patient discharged in a good general condition.

\section{REFERENCES}

1. Jurkovich GI. Injury to the duodenum and pancreas. In: Feiciano DV, Moore EE, Mattox KL, editors. Trauma, $3^{\text {rd }}$ ed. Appleton \& Lange; Stamford CT: 1996;573-694.

2. Guo-ging CHEN, Hua YANG. Management of duodenal trauma. Chin J Traumatol 2011;14(1):61-4. 
3. Mindelzum RE, Jeffrey RB. The acute abdomen: current CT imaging techniques. Semin Ultrasound CT MR 1999;20(2):63-7.

4. Jayaraman MV, Mayo-Smith WW, Movson JS, et al. CT of the duodenum: an overlooked segment gets its due. Radiographics 2001;21(1):S147-S160.

5. Brotman S, Cisternion S, Myers RAM, et al. A test to help diagnosis of rupture in the injured duodenum. Injury 1981;12(6):464-5.

6. Moore EE, Cogbill TH, Malangoni MA, et al. Organ injury scaling, II: pancreas, duodenum, small bowel, colon, and rectum. J Trauma 1990;30(11):1427-9.
7. Degiannis E, Boffard K. Duodenal injuries. Br J Surg 2000;87(11):1473-9.

8. Ginzburg E, Carrillo EH, Sosa JL, et al. Pyloric exclusion in the management of duodenal trauma: is concomitant gastrojejunostomy necessary? Am Surg 1997;63(11):964-6.

9. Asensio JA, Feliciano DV, Britt LD, et al. Management of duodenal injuries. Curr Probl Surg 1993;30(11):102393.

10. Ivatury RR, Nassoura ZE, Simon RJ, et al. Complex duodenal injuries. Surg Clin North Am 1996;76(4):797812. 